\title{
Atrial functional mitral regurgitation - a reversible valvulopathy
}

\author{
Pankaj Mathur $^{1 *}$, Thomas Joseph ${ }^{1}$, Srikanth Vallurupalli² and Hakan Paydak ${ }^{2}$ \\ ${ }^{1}$ Department of Internal Medicine, University of Arkansas Medical Sciences, USA \\ ${ }^{2}$ Division of Cardiology, Department of Internal Medicine, University of Arkansas Medical Sciences, USA
}

\begin{abstract}
Atrial fibrillation is a common clinical arrhythmia seen worldwide in clinical practice. Atrial fibrillation associated functional mitral regurgitation (AFMR) has been described recently in several studies. Here we describe a patient with AFMR which improved with achievement of sinus rhythm by cardioversion and anti-arrhythmic therapy. The general internist needs to be aware of this condition and more studies are needed to define the evidence-based management of this condition.
\end{abstract}

\section{Background}

Atrial fibrillation is the most common tachyarrhythmia encountered in the clinical practice worldwide [1]. Prolonged atrial fibrillation promotes remodeling of the atria resulting in atrial dilation which in turn can affect valvular function. Mitral regurgitation (MR) resulting from such changes in the atria has been described in patients with structurally normal valves and atrial fibrillation. One term to describe this phenomenon is "atrial functional mitral regurgitation (AFMR)". Achievement of sinus rhythm appears to ameliorate the severity of mitral regurgitation but some patients may require surgery. The role of medical management in these patients is unknown. In this report, we describe a patient with AFMR which improved with achievement of sinus rhythm by cardioversion and anti-arrhythmic therapy.

\section{Case History}

A 58 year old male with past medical history of gastroesophaeal reflex disease, actinic keratosis and obstructive sleep apnea presented to the outpatient cardiology clinic for incidentally discovered atrial fibrillation by electrocardiography. He denied chest pain, shortness of breath, palpitations, or edema. His physical examination was within normal limits except for an irregulary irregular pulse and holosystolic murmur at the apex. Transthoracic echocardiogram revealed a structurally normal mitral valve with moderate mitral regurgitation (Figure 1a), moderate tricuspid regurgitation, mildly dilated left atrium and normal left ventricular ejection fraction. Based on a discussion of risks and benefits, he elected to a rhythm control strategy. He was started on rivaroxaban and underwent successful electrical cardioversion to sinus rhythm. He was started on amiodarone initially and was switched to flecainide after negative workup for ischemic heart disease. Repeat echocardiography revealed a significant reduction in severity of mitral regurgitation (Figure 1b). At 18 months of follow-up, he remains in sinus rhythm and his mitral regurgitation remains mild in severity.

\section{Discussion}

AFMR occurs in the presence of normal left ventricular function and a structurally normal mitral valve [2-4]. The pathogenesis of atrial functional MR was initially attributed mainly to annular dilatation

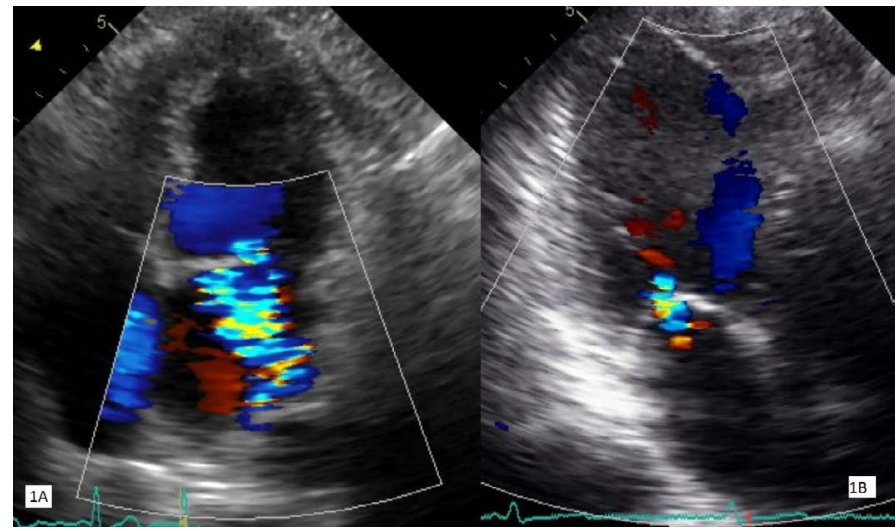

Figure 1a, 1b. Atrial functional mitral regurgitation before and after medical management.

[4]. However, several mechanisms (sometimes synergistically) are instrumental in causing significant AFMR. Loss of the appropriately timed atrial contraction in AF by itself impairs coaptation of mitral valve leaflets [5]. Several other factors including contractile dysfunction of mitral annulus, disruption of the annular saddle shape, and atriogenic posterior mitral valve tethering also contribute to AFMR [6-8]. Of these, atriogenic posterior mitral valve tethering is likely a significant contributor and is well studied [8]. As the left atrium enlarges, the posterior wall of the left atrium extends behind the posterior wall of the LV resulting in a) displacement of the posterior mitral annulus behind the LV inlet and b) displacement of the basal posterior LV wall forward towards the LV outflow [8]. This causes tethering of the posterior leaflet and leaflet malcoaptation resulting in AFMR.

*Correspondence to: Pankaj Mathur, Assistant Professor, Department of Internal Medicine, University of Arkansas Medical Sciences, UAMS Medical Center, 4301 W Markham Street, Little Rock, Arkansas 72205, USA, E-mail: PMathur@uams.edu

Received: May 22, 2018; Accepted: July 11, 2018; Published: July 14, 2018 
Initial studies on AFMR suggested that the severity of mitral regurgitation was rarely severe enough to warrant surgery [3]. However, later several reports suggested that AFMR, is sometimes severe enough to warrant mitral valve surgery $[9,10]$. It appears that some patients with chronic atrial fibrillation may also develop severe atrial remodeling which in turn causes significant AFMR. The incidence of at least moderate AFMR was as high as $6.4 \%$ in a series of patients referred for AF ablation [4]. The challenge is however in predicting which patient is likely to develop AFMR and who would benefit from an aggressive rhythm control strategy.

Since the major driver of AFMR is persistent AF, sinus rhythm (if achieved before significant remodeling occurs) should theoretically ameliorate this condition. The best evidence that achievement of sinus rhythm improves AFMR comes from a large series of patients who underwent atrial fibrillation ablation [4]. In this series, patients who achieved and maintained sinus rhythm with atrial fibrillation ablation had significant reduction in left atrial size and annular dimension and lower rates of significant AFMR. The effect of AFMR on outcomes after AF ablation are also being increasingly recognized. Presence of AFMR represents significant atrial remodeling and predicts recurrence after AF ablation $[11,12]$. The effect of significant AFMR on left atrial pressure is another factor that likely triggers recurrence after ablation.

The current case illustrates that the severity of AFMR can be reduced by a simple strategy of cardioversion and anti-arrhythmic therapy. Over the past decade, the treatment of atrial fibrillation has been heavily influenced by the results of the AFFIRM trial, which showed that rate control was non inferior to rhythm control in an elderly population (mean age 69 years) [13]. These results were influenced by lower use of warfarin in the rhythm control group and side effects of anti-arrhythmic drugs. However, application of a blanket strategy of rate control in young patients may subject them to long term remodeling of the atria and resultant valvular dysfunction. As the prevalence of AF increases and patients with $\mathrm{AF}$ live longer, the incidence of AFMR is expected to increase as well. Based on available data, achievement of sinus rhythm should be strongly considered in patients with at least moderate AFMR though this approach has not been tested in a prospective trial. In addition, other measures to reduce left atrial pressure and related remodeling such as aggressive treatment of hypertension and achieving ideal weight should be considered.
This case illustrates a reversible cause of mitral regurgitation. Clinicians should be aware of the effect of chronic atrial fibrillation on left atrial structure, which in some patients may cause significant AFMR.

\section{References}

1. January CT, Wann LS, Alpert JS, Calkins H, Cigarroa JE, et al. (2014) AHA/ACC/HRS guideline for the management of patients with atrial fibrillation: executive summary: a report of the American College of Cardiology/American Heart Association Task Force on practice guidelines and the Heart Rhythm Society. Circulation 130: 2071-2104. [Crossref]

2. Kihara T, Gillinov AM, Takasaki K, Fukuda S, Song JM, et al. (2009) Regurgitation associated with mitral annular dilation in patients with lone atrial fibrillation: an echocardiographic study. Echocardiography 26: 885-889. [Crossref]

3. Zhou X, Otsuji Y, Yoshifuku S, Yuasa T, Zhang H, et al. (2002) Impact of atrial fibrillation on tricuspid and mitral annular dilatation and valvular regurgitation. Circ $J$ 66: 913-916. [Crossref]

4. Gertz ZM, Raina A, Saghy L, Zado ES, Callans DJ, et al. (2011) Evidence of atria functional mitral regurgitation due to atrial fibrillation: reversal with arrhythmia control. J Am Coll Cardiol 58: 1474-1481. [Crossref]

5. David D, Michelson E, Naito M, Chen CC, Schaffenburg M, et al. (1983) Diastolic "locking" of the mitral valve: the importance of atrial systole and intraventricular volume. Circulation 67: 640-645

6. Machino-Ohtsuka T, Seo Y, Ishizu T, Sato K, Sugano A, et al. (2016) Novel Mechanistic Insights into Atrial Functional Mitral Regurgitation- 3-Dimensional Echocardiographic Study. Circ J 80: 2240-2248.

7. Ring L, Dutka DP, Wells FC, Fynn SP, Shapiro LM, et al. (2014) Mechanisms of atria mitral regurgitation: Insights using 3D transoesophageal echo. Eur Heart J Cardiovasc Imaging 15: 500-508.

8. Silbiger JJ (2013) Novel pathogenetic mechanisms and structural adaptations inischemic mitral regurgitation. J Am Soc Echocardiogr 26: 1107-1117.

9. Kilic A, Schwartzman DS, Subramaniam K, Zenati MA (2010) Severe functional mitral regurgitation arising from isolated annular dilatation. Ann Thorac Surg 90: 1343-1345.

10. Takahashi Y, Abe Y, Sasaki Y, Bito Y, Morisaki A, et al. (2015) Mitral valve repair for atrial functional mitral regurgitation in patients with chronic atrial fibrillation. Interact Cardiovasc Thorac Surg 21: 163-168.

11. Qiao Y, Wu L, Hou B, Sun W, Zheng L, et al. (2016) Functional mitral regurgitation: predictor for atrial substrate remodeling and poor ablation outcome in paroxysmal atrial fibrillation. Medicine (Baltimore) 95: e4333.

12. Gertz ZM, Raina A, Mountantonakis SE, Zado ES, Callans DJ, et al. (2011) The impact of mitral regurgitation on patients undergoing catheter ablation of atrial fibrillation. Europace 13: 1127-1132.

13. Wyse DG, Waldo AL, DiMarco JP, Domanski MJ, Rosenberg Y, et al. (2002) Atrial Fibrillation Follow-up Investigation of Rhythm Management (AFFIRM) Investigators. A comparison of rate control and rhythm control in patients with atrial fibrillation. $N$ Engl J Med 347: 1825-1833.

Copyright: (C2018 Mathur P. This is an open-access article distributed under the terms of the Creative Commons Attribution License, which permits unrestricted use, distribution, and reproduction in any medium, provided the original author and source are credited. 